\title{
Estudio comparativo de dos dispositivos intrauterinos, la espiral delta y la espiral de Lippes D colocados a pacientes postparto
}

\author{
Dres.: Saavedra Beltrán*, Cynthia Waszak**
}

\section{INTRODUCCION}

La inserción del DIU en el periodo de postparto presenta las siguientes ventajas: 1) colocación fácil, debido a la dilatación cervical; 2) ocultamiento de la pérdida de sangre por los loquios (5); 3 ) alta motivación por la anticoncepción inmediatamente después del parto, y 4) la conveniencia de poder insertar el DIU mientras la mujer está en el hospital (4). Esta última ventaja es especialmente importante en los países en desarrollo, donde el contacto de la mujer con la profesión médica es limitada. La elección del dispositivo intrauterino como método anticonceptivo en el período postparto es conveniente para la mujer lactante, particularmente porque los DIU tienen poco o ningún efecto sobre la lactancia (2) y a su vez ésta no afecta la forma en que actúan los DIU (3).

Por otra parte, el principal inconveniente de la inserción de DIU durante el postparto es la muy elevada tasa de expulsión debida a la involución uterina.

Hospital de Juan Noé, Arica, Chile

* Family Health International, Research Triangle Park, NC 27709, USA.
Previos estudios de postparto han demostrado a este respecto tasas de expulsión que oscilan desde 17,5 hasta 41,3 $(1,6)$. En un esfuerzo por reducir estas tasas, el IFRP ha diseñado un modelo modificado de la espiral de Lippes D y del dispositivo $\mathrm{T}$ de cobre.

El presente estudio forma parte de un ensayo multi-institucional destinado a comparar la espiral de Lippes D estándar con el modelo modificado, la espiral Delta, con respecto a tasas de expulsión registradas.

\section{MATERIAL Y METODOS}

Desde abril de 1979 hasta octubre de 1980 inclusive, se insertó la espiral Delta y la espiral de Lippes D en 259 pacientes postparto del Hospital de Juan Noé, de Arica, Chile. Los dispositivos se insertaron en forma manual diez minutos después de expulsada la placenta. Los datos se anotaron en formularios estandarizados confeccionados para obtener información médica y sociodemográfica. Se analizaron los datos de cada uno de los dos grupos. En el grupo de la espiral Delta participaron 136 mujeres y en el de la espiral de Lippes D participaron 123. Las integrantes de los dos grupos eran simila- 
res con respecto a la edad, paridad y educación. Se anticipó que las mujeres regresaran al 1er. 3er. y 6o. mes después de insertado el DIU para exámenes de continuación.

Los dispositivos utilizados fueron la espiral de Lippes D y la espiral modificada (espiral Delta). A la espiral Delta se le agregaron tres suturas con catgut en el segmento transversal para que el DIU encajara en el endometrio, evitándose así su expulsión durante la involución.

\section{RESULTADOS}

En el Cuadro No. 1 se presentan las complicaciones y trastornos en el momento de la inserción y posteriormente. Durante la inserción se produjo una laceración que requirió sutura en el grupo de la espiral de Lippes D. Una mujer del grupo de la espiral Delta se quejó de leves dolores pélvicos durante la inserción.

La incidencia de dismenorrea y flujo intermenstural, goteo $y$ dolores infor-

\section{Cuadro No. 1}

\section{COMPLICACIONES/TRASTORNOS DURANTE LA INSERCION Y EL SEGUIMIENTO*}

\begin{tabular}{|c|c|c|c|c|}
\hline & \multicolumn{2}{|c|}{$\begin{array}{l}\text { Espiral Delta } \\
(N=136)\end{array}$} & \multicolumn{2}{|c|}{$\begin{array}{l}\text { Espiral de Lippes D } \\
\qquad(N=123)\end{array}$} \\
\hline & No. & 옹 & No. & $\%$ \\
\hline \multicolumn{5}{|l|}{$\begin{array}{l}\text { Complicaciones/trastornos } \\
\text { durante la inserción }\end{array}$} \\
\hline Laceración que requiere sutura & 0 & 0,0 & 1 & 0,8 \\
\hline Leves dolores pélvicos & 1 & 0,7 & 0 & 0,0 \\
\hline \multicolumn{5}{|l|}{$\begin{array}{l}\text { Complicaciones/trastornos } \\
\text { durante el seguimiento }\end{array}$} \\
\hline Dismenorrea & 23 & 16,9 & 23 & 18,9 \\
\hline \multicolumn{5}{|l|}{ Trastornos intermenstruales: } \\
\hline Pérdidas sangu íneas & 5 & 3,7 & 2 & 1,6 \\
\hline Goteo ("spotting") & 4 & 2,9 & 2 & 1.6 \\
\hline Dolor & 7 & 5,1 & 4 & 3,3 \\
\hline \multicolumn{5}{|l|}{ Inflamación pélvica } \\
\hline Anexitis & 1 & 0,7 & 0 & 0,0 \\
\hline Endometritis & 3 & 2,2 & 5 & 4,1 \\
\hline Otras & 2 & 1,5 & 2 & 1,6 \\
\hline Hospitalización & 1 & 0,7 & 2 & 1,6 \\
\hline
\end{tabular}

* Puede haberse notificado más de un trastorno/complicación por mujer. 
mados durante visitas posteriores fue similar en los dos grupos.

A tres mujeres (2.2\%) del grupo de la espiral Delta se les diagnosticó endometritis. Una mujer (0.7\%) volvió a ser hospitalizada, y se le extrajo el dispositivo debido a anexitis. En el grupo de la espiral de Lippes $D$, cinco mujeres
(4.1\%) presentaron endometritis $\mathrm{y}$ una de ellas tuvo que volver al hospital. Otra de las mujeres debió ser hospitalizada otra vez por tener hemorragias (0.8\%).

En el Cuadro No. 2 se presentan los eventos de la tabla de vida y las tasas de terminación. Las tasas corresponden

Cuadro No. 2

TASAS CUMULATIVAS APROXIMADAS DE LA TABLA DE VIDA

Espiral Delta

$\langle\mathrm{N}=136\rangle$
Espiral de Lippes D

$(N=123)$

\section{Embarazo accidental}

1 mes

3 meses

Explusión

1 mes

3 meses
$0,0+0,0$

$0,0+0,0$

$11,7+2,8$

$15,3+3,2$
$0,0+0,0$

$1,2+1,2$

Extracción por dolor/pérdidas
1 mes
3 meses
$0,0+0,0$
$0,0+0,0$
$1,0+1,0$
$0,0+0,0$

Extracció por otras razones médicas

1 mes

$1.6+1.2$

$1,9+1,3$

3 meses

$1.6+1,2$

$1,9+1,3$

Continuación

1 mes

86,8

78,5

3 meses

81,6

68,1

Seguimiento**

1 mes

94.9

94.3

3 meses

77.0

* Significativa a $=0,05$

* Significativa a $=0,01$

** Se entiende por tasa de seguimiento el porcentaje de mujeres que no se han dado por concluidas y que regresan para exámenes de control. 
a un período de tres meses en lugar de seis debido a la baja tasa de seguimiento existente a los seis meses. En el lapso de tres meses la tasa de expulsión de la espiral Delta de 15,3 fue inferior a la de 28,3 correspondiente a la espiral de Lippes $D(p \leq 0,05)$. En ambos grupos, las expulsiones fueron más numerosas en el primer mes que en cualquier mes subsiguiente, como era de esperar.

Las tasas de otras terminaciones fueron similares en los dos grupos. En tres meses, la tasa de embarazo accidental fue de 0.0 para el grupo de la espiral Delta y de 1,2 para el grupo de la espiral de Lippes D. En el mismo período, la tasa de extracción debida a pérdidas sanguíneas $y$ dolores fue de 1,0 en las usuarias de la espiral Delta y de 0,0 en las usuarias de la espiral de Lippes D; las tasas de extracción por otras razones médicas fueron de 1,6 y de 1,9, respectivamente. Al final de tres meses de seguimiento, la tasa de continuación general fue de 81,6 en las usuarias de la espiral Delta y de 68,1 en las usuarias de espiral de Lippes $D(p \leq 0,01)$.

\section{DISCUSION}

Casi todos los niveles de comportamiento fueron similares en ambos dispositivos. Las tasas de embarazo accidental, extracción debida a dolores/ pérdidas sanguíneas y extracción por otras razones médicas, no fueron estadísticamente diferentes en los dos grupos y tampoco se registraron diferencias en el número de mujeres de uno y otro grupo que notificaron problemas intermenstruales o menstruales o que presentaron inflamaciones pélvicas.

Sin embargo, el principal objetivo del presente estudio fue el de averiguar las tasas de expulsión. La espiral Delta se diseñó para reducir las tasas de expulsión, como efectivamente se comprobó en el estudio. Aunque en las usuarias de la espiral Delta las tasas de expulsión fueron más altas que las de las mujeres con un intervalo entre el parto y la inserción, éstas fueron significativamente más bajas que las de la espiral de Lippes $D$ en una población similar de pacientes postparto. Las tasas de expulsión encontradas en este estudio son comparables a las comunicadas en un trabajo publicado anteriormente sobre estudios preliminares de dispositivos Delta. En dicha comunicación, la tasa de expulsión de espiral Delta a los seis meses fue de 17.4 (313 inserciones), en comparación con una tasa de expulsión de 24 para la espiral de Lippes D (285 inserciones) en los ensayos multi-institucionales (4).

\section{CONCLUSION}

A pesar de los inconvenientes de la inserción durante el postparto, uno de los obstáculos principales es la elevada tasa de expulsión. Se han introducido modificaciones a la espiral de Lippes D (espiral Delta) a fin de reducir esta alta tasa de expulsión. Se agregaron suturas a los dispositivos intrauterinos para que encajaran en el endometrio, evitándose su expulsión durante la involución uterina. En el presente estudio las tasas de expulsión correspondientes a ambos grupos fueron más elevadas que en las de estudios de intervalo; la tasa de expulsión de la espiral Delta fue, sin embargo, apreciablemente más baja que la de la espiral de Lippes D sin modificaciones.

\section{RESUMEN}

Los dispositivos intrauterinos (DIU), como método anticonceptivo de las mujeres que han tenido alumbramientos recientes, se han utilizado en forma limitada debido a las altas tasas de expulsión relacionadas con su colocación en el período de postparto. En un esfuerzo por corregir dicho problema, el Progra- 
ma Internacional de Investigaciones en Fertilidad (IFRP) ha introducido modificaciones en dos tipos de DIU, a saber, la espiral de Lippes $D$ y el dispositivo $T$ de cobre 220C. La modificación consiste en el agregado de tres suturas de catgut No. 2 que se desechan biológicamente al completarse el proceso de involución. El estudio se diseñó con el objeto de poner a prueba la espiral de Lippes D modificada (espiral Delta) y compararla con el Lippes Loop D estándar. La espiral Delta se insertó en 136 mujeres del Hospital de Juan Noé en Arica, Chile.
En cada uno de los casos, la inserción ocurrió dentro de los 10 minutos después de la expulsión de la placenta. El estudio confirmó la expectativa de que la tasa de expulsión de las espirales Delta sería significativamente más baja que la de las espirales sin modificar, pues las tasas fueron de 15,8 en el caso de las usuarias de espirales Delta y 28.3 para las usuarias de espiral de Lippes D $(p \leq 0,05)$. A los tres meses, las tasas de continuación de uso de los dos grupos fueron de 81,6 y de 68,1 , respectivamente.

\section{COMPARATIVE STUDY OF TWO INTRA-UTERINE DEVICES, THE DELTA LOOP AND THE LIPPES D LOOP INSERTED POST-PARTUM}

\section{SUMMARY}

Intra-uterine devices (IUD) have not been used widely by patients with recent deliveries due to the high expulsion rates associated with post-partum insertion. In an effort to address the problem, the International Fertility Research Program (IFRP) introduced modifications in two types of IUD: the Lippes D loop and the 220c Copper T. The modification consisted of three No. 2 Catgut sutures added to the IUD's to biodegrade as the process of involution finishes.

\section{BIBLIOGRAFIA}

1. AZNAR R, REYNOS L, MONTEMAYOR. $G$ y GINER J. Post-placental insertion of IUDs. Contracep Deliv Syst. 1: 1431980.

2. BADRAOUI M, HEFNAWI F, BAHGATR. FAWZI G, EL GAALI O. Contraception during lactation. Reproduction 6:1, 1982.

3. COLE L, MCCANN M, HIGGINS J y WASZAK C. The effects of breast-feeding on IUD performance. Trabajo presentado en la 109a. Reunión Anual de la American Public
The purpose of this study was to test the modified Lippes D (Delta loop) and compare it with the standard Lippes D loop. The Delta loop was inserted in 136 women at Juan Noé Hospital in Arica, Chile. In every case insertion took place within ten minutes following expulsion of the placenta.

The study confirmed that the rate of expulsion of the Delta loop was significantly lower than that of the standard loop (15.8 vs. $28.3, p-0,05)$. After three months, the continuation rates were 81.6 and 68.1 respectively.

Health Association, Los Angeles, 1-5 de noviembre de 1981.10 págs.

4. EDELMAN DA, GOLDSMITH A y JD SHELTON. Postpartum contraception. Int J. Gynaecol Obstet 19: 305, 1981.

5. LAUFE L, WHEELER R y $P$ FRIEL. Modification of intrauterine devices for postpartum insertion. Lancet, 21 de abril de 1979, págs. 853-854.

6. OMS, Comparative multi-centre trial of three IUDs inserted immediately following the delivery of the placenta. Contraception 22 (1): 9 (year?). 